\title{
Molecular Dynamics Study of the Effect of Electrostatic Interactions on the Biphenyl Structure in the Active $\mathrm{HNO}_{3}$ Solvent
}

\author{
Kholmirzo Kholmurodov*,1,2 Alena Chulkova $^{2}$ and Kenji Yasuoka ${ }^{3}$ \\ ${ }^{1}$ Laboratory of Radiation Biology, Joint Institute for Nuclear Research, 141980, Dubna, Russia \\ ${ }^{2}$ International University “Dubna”, 141980, Dubna, Moscow Region, Russia \\ ${ }^{3}$ Department of Mechanical Engineering, Keio University, Yokohama 223-8522, Japan
}

\begin{abstract}
An analysis of the molecular dynamics (MD) of the biphenyl molecular complex in an acidic solution has been performed. The aim of this work is to study the influence of the electrostatic (Coulomb) forces on the dynamical and structural behavior of the biphenyl molecules interacting with the $\mathrm{HNO}_{3}$ solvent. The temperature and energy characteristics and the phase transition of the biphenyl molecule point to the existence of a critical charge number for the $\mathrm{HNO}_{3}$ acid particles that control the interaction process.
\end{abstract}

Keywords: Molecular dynamics simulations, biphenyl molecule, active solvent, phase transition.

\section{INTRODUCTION}

In recent years, there has been a great interest in studying the chemical and physical properties of the biphenyl molecule and its various compounds, which is greatly motivated by the needs of the environmental science concerning both research and application aspects[1-3]. As of today, biphenyls along with dioxins, carbon and nitrogen monoxides (CO and $\mathrm{NO}$ ), dichloro-diphenyl-trichloroethane (DDT), and some others, are a constituent part of the active chemical reagents which form a main basis for industrial waste or environmental pollution. Biphenyls, for example, are able to form chemical bonds with soil minerals, and, hence, can influence the structure of soil and its physical and chemical properties [4-6].

Biphenyl (phenylbenzene, diphenyl) is an aromatic hydrocarbon with a distinctively pleasant smell; its molecular formula is $\left(\mathrm{C}_{6} \mathrm{H}_{5}\right)_{2}$ and its molecular mass is 154.20 a.m.u. It is an organic compound that forms colorless crystals; the melting and boiling temperatures are correspondingly $70.5^{\circ} \mathrm{C}$ and $254.5^{\circ} \mathrm{C}$; it is easily soluble in spirits, ether, benzene, and many organic solvents, but not soluble in water. It is worth noting that biphenyl molecule possesses a rotational degree of freedom around its central bond; the benzene rings in biphenyl have to be coplanar for crystals, but in solvent media they are inclined relatively to each other.

Modeling biphenyl's structural properties in its different complexes with solvents and ionic reagents is a task of a great interest - in material and environmental sciences, biochemistry, and biochemical informatics [2,6]. Studies on biphenyl's structural peculiarities and its phase transition behavior can provide valuable information that is needed,

*Address correspondence to this author at the Joint Institute for Nuclear Research, Dubna International University, 141980, Dubna, Moscow Region, Russia; Tel: (+7 49621) 62872; Fax: (+7 49621) 65948;

E-mail: mirzo@jinr.ru firstly, for environmental research, science, and applied industry $[2,3]$. The biphenyl molecule has been studied from the point of view of its application in pharmacology and drug delivery [4]; for the tasks of chromatography [1-3]; in the development of an enzyme catalysis and protein folding model $[7,8]$ etc.

In the present work, based on the molecular dynamics (MD) method, we have simulated biphenyl's temperature energy characteristics and phase transition behavior in interactions with active nitric acid $\left(\mathrm{HNO}_{3}\right)$. It is well known that $\mathrm{HNO}_{3}$ is a highly corrosive and toxic, strong acid; all biphenyl salts - i.e., nitrates - are obtained as a result of $\mathrm{HNO}_{3}$ acting on metals, oxides, hydroxides, and carbonates $[1,2,6]$. All nitrates have to be well soluble in water; their solvents provide an oxidizing reaction. The specific effect of the nitric acid on the biphenyl molecule exhibits a number of nontrivial peculiarities, followed by the active involvement of an aromatic compound that has a carbonyl group inside. For example, the biphenyl carbon acid will go through a transition state, the energy of which has been strongly affected by the Coulomb forces between the carboxyl group and nitronium cation. The interaction forces and their nature (attractive or repulsive) depend on the structural configuration of the biphenyl carbon acid [1-6,9]. In this work, we investigate the dynamical and structural properties of the biphenyl molecule interacting with the active solvent $\mathrm{HNO}_{3}$. We aimed to elucidate the effect of the electrostatic (Coulomb) forces on the biphenyl $+\mathrm{HNO}_{3}$ system under different interaction modes, which are controlled by a charge number of the solvent particles.

\section{MATERIALS AND METODS}

The molecular dynamics (MD) simulations have been performed using the DL_POLY code, which was developed by the Molecular Simulation Group at Daresbury Laboratory (England) under the support of the Research Council for the Engineering and Physical Sciences (project CCP5 for the simulation of condensed phases). DL_POLY is a general- 


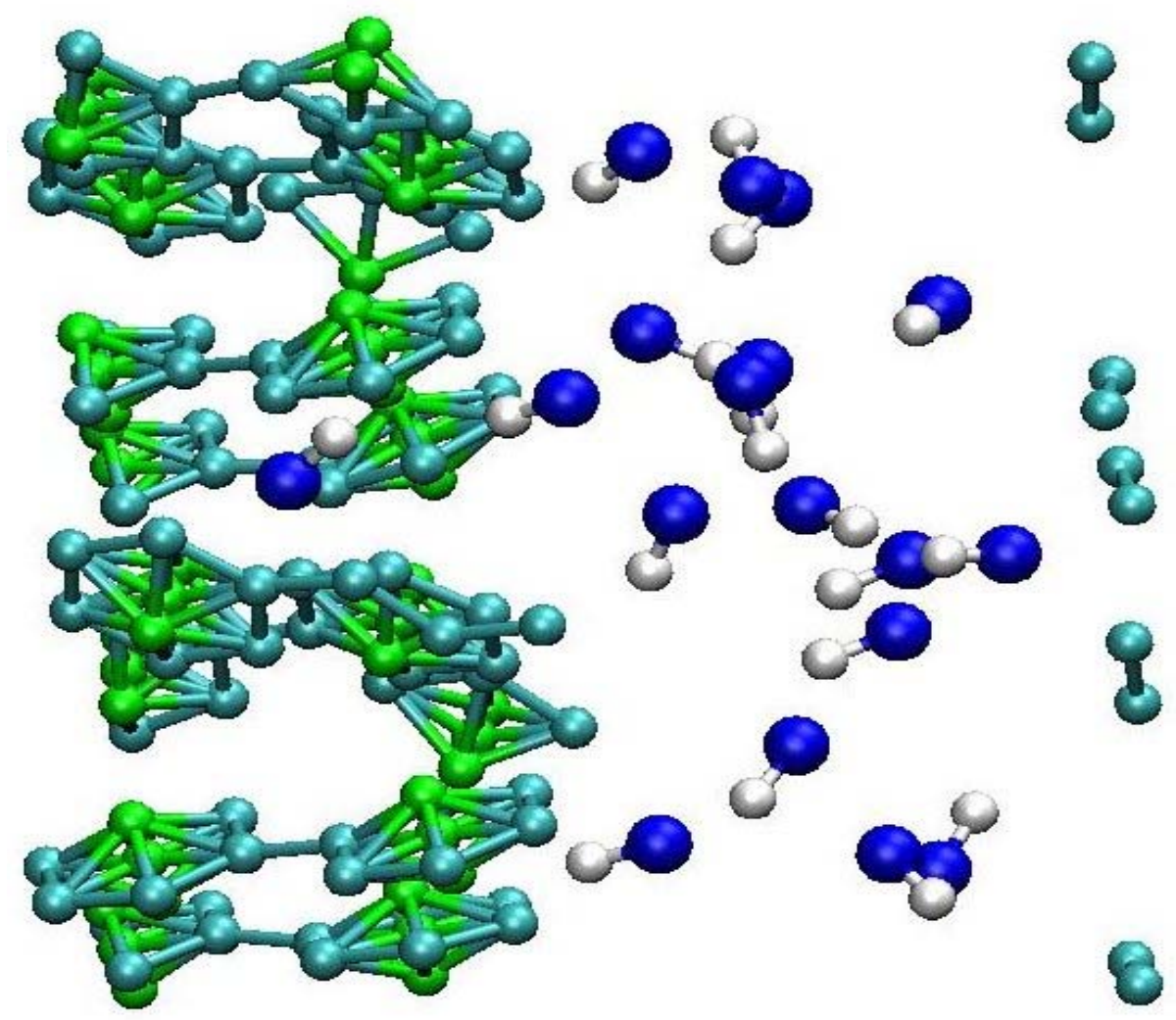

Fig. (1). The initial configuration of biphenyl molecules in the acid solvent $\mathrm{HNO}_{3}$.

purpose MD simulation package developed by W. Smith, T.P. Forester and I.T. Todorov [10,11]. We have employed DL_POLY version 2.19; the initial geometry of the biphenyl molecule was chosen from the database of the program package at:

http://www.cse.scitech.ac.uk/ccg/software/DL_POLY/

The initial configuration of the biphenyl molecule in the active solvent is shown in Fig. (1). Periodic boundary conditions have been applied for all directions. To build up the system's starting configuration, we employed the utilities and interfaces which are provided by the DL_POLY package for the visualization purposes and analysis of the results.

The configuration energy of the molecular model is represented as a sum of the energies of the bonding $\left(\mathrm{E}_{\mathrm{val}}\right)$ and non-bonding $\left(\mathrm{E}_{\mathrm{nb}}\right)$ interactions:

$\mathrm{E}=\mathrm{E}_{\mathrm{val}}+\mathrm{E}_{\mathrm{nb}}$.

The energy of the valence (bonding) interactions $E_{v a l}$ is given by the following formula:

$\mathrm{E}_{\mathrm{val}}=\mathrm{E}_{\mathrm{imb}}+\mathrm{E}_{\mathrm{ang}}+\mathrm{E}_{\mathrm{dih}}+\mathrm{E}_{\mathrm{inv}}$,

where $\mathrm{E}_{\mathrm{imb}}$ is the energy of intermolecular bonds, $\mathrm{E}_{\mathrm{ang}}$ is the energy of angular bonds, $E_{\mathrm{dih}}$ is the energy of dihedral bonds, and $\mathrm{E}_{\mathrm{inv}}$ is inversion energy.

The energy of the non-valence (non-bonded) interactions is a sum of the energies of the van-der-Waals (vdW), electrostatics (Coulomb), and hydrogen bonds:

$\mathrm{E}_{\mathrm{nb}}=\mathrm{E}_{\mathrm{VdW}}+\mathrm{E}_{\mathrm{coul}}+\mathrm{E}_{\mathrm{hb}}$.

In the biphenyl molecule, each phenyl ring is represented as a rigid body; we have simulated 8 biphenyl molecules with the total number of atoms being 144. In the centre of each phenyl ring, there are three massless charged sites, which guarantees the existence of a quadrupole moment of the ring (Fig. (2a, b)). The active solvent is a complex of the $\mathrm{HNO}_{3}$ particles, which are represented as bi-atomic molecules (Fig. (2c)), with charges that are equal and of opposite signs. The charge on each site has been chosen as $\mathrm{q}^{*}=\mathrm{qe}$, where e is a charge unit (the proton charge). The charge number $q$ varied during the simulations to take all possible values in the interval $[0,1]$ with a step $\Delta q=0,01$. The values of the masses and charges of particles used in the MD simulations are presented in Table 1 , where $m_{e}=1,66 \mathrm{x}$ $10^{-27} \mathrm{~kg}$ is an atomic unit of mass (a.u.m.); e $=1,6 \times 10^{-19} \mathrm{Q}$ is the proton charge.

During the MD simulations, the following three potential types, which represent a topology of the molecular field for the biphenyl $+\mathrm{HNO}_{3}$ system, were used:

(1) Power series potential (nm),

(2) Buckingham potential (buck),

(3) Lennard - Jones potential (lj).

The interaction parameters and potential functional forms are shown in Tables (2a-c). The computer simulations have been performed for a MD cell of a volume $V=(13,68 \AA)^{3}$, under the energy and temperature control at $\mathrm{T}=250 \mathrm{~K}$. The number of biphenyl molecules was $\mathrm{N}=8$ (144 atoms); the active solvent $\left(\mathrm{HNO}_{3}\right)$ consisted of $\mathrm{N}=18$ molecules, 36 sites, which are constrained within a flexible bond with a length of about $1 \AA$. The integration of the equations of motion was performed using the Verlet integration scheme in quaternion. The integration step was 1 fs (femtosecond); a 
(a)

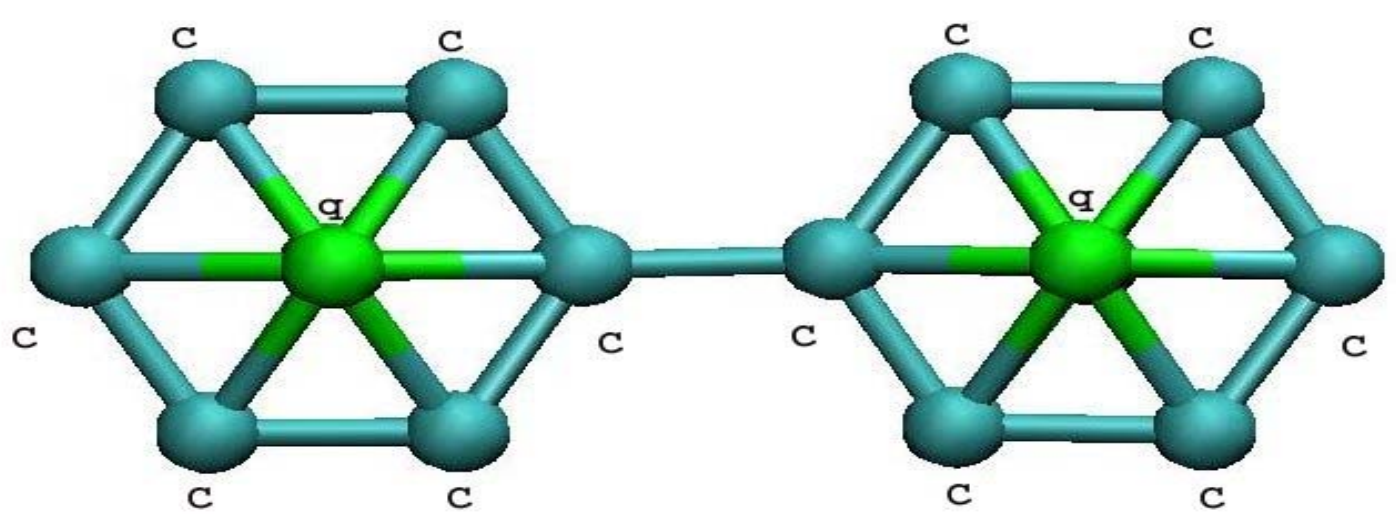

(b)

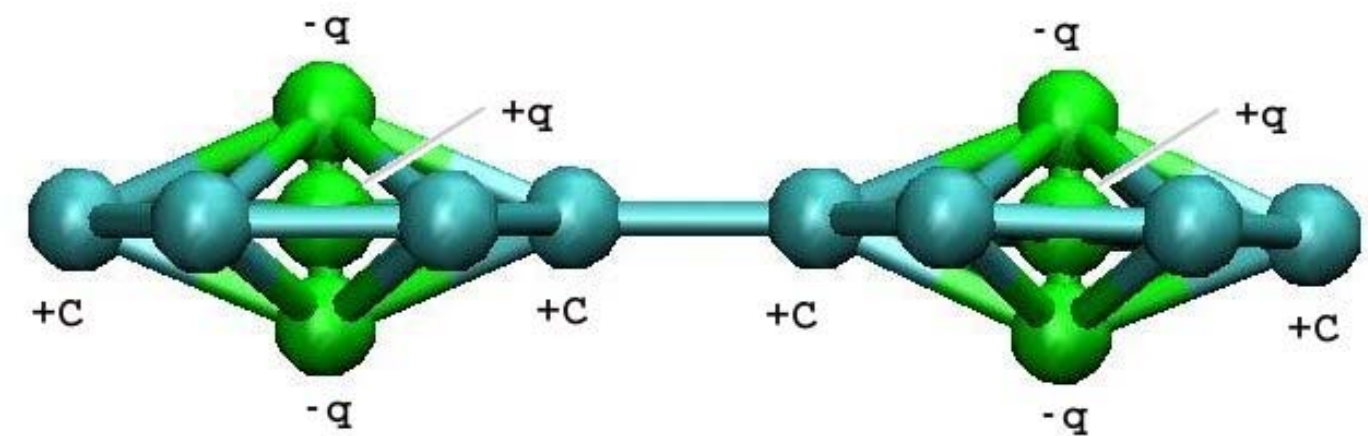

(c)

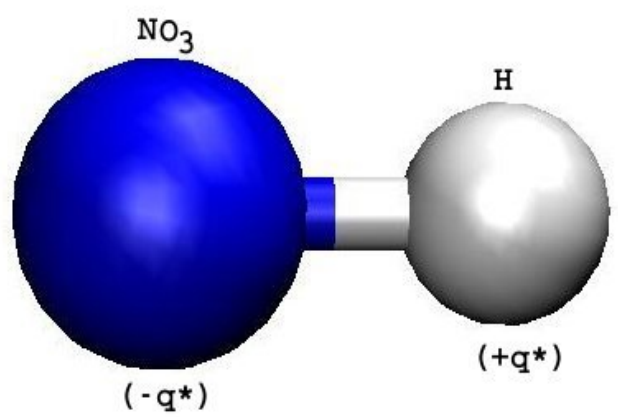

Fig. (2). Biphenyl configuration (side view (a) and top view (b)). Two aromatic rings of carbon atoms $\mathrm{C}$ and a charge site $\mathrm{q}$ in the center are displayed. In the center of each phenyl ring, there are three massless charges q. A snapshot of the solvent $\mathrm{HNO}_{3}$ molecule surrounding biphenyl is presented (c). A negative site $\mathrm{NO}_{3}$ is shown blue; a hydrogen atom $\mathrm{H}$ is shown white.

Table 1. The Values of the Masses and Charges in the Model System Biphenyl+HNO

\begin{tabular}{|c|c|c|}
\hline Atom & Mass number $\mathbf{m}\left(\mathbf{m}_{\mathbf{e}}, \mathbf{a . u . m .}\right)$ & Charge number q (e, proton charge) \\
\hline \hline $\mathrm{C}$ & 13.02 & +0.15 \\
\hline $\mathrm{Q}$ & 0. & -2.25 \\
\hline $\mathrm{Q}$ & 0. & +3.61 \\
\hline $\mathrm{NO}_{3}$ & 62.005 & $0,-0.01,-0.02,-0.03, \ldots,-1$ \\
\hline $\mathrm{H}$ & 1.008 & $0,+0.01,+0.02,+0.03, \ldots,+1$ \\
\hline
\end{tabular}


Table 2a. The Potential and Parameters for the Atomic Pair C-q

\begin{tabular}{|c|c|c|c|c|c|c|c|}
\hline Atomic pair & Potential & Functional Form & Parameters & $\mathbf{E}_{0}, \mathrm{~kJ} / \mathrm{mol}$ & $\mathbf{N}$ & $\mathbf{m}$ & $\mathbf{r}_{0}, \AA$ \\
\hline $\mathrm{C}-\mathrm{q}$ & $\mathrm{nm}$ & $U(r)=\frac{E_{0}}{(n-m)}\left[m\left(\frac{r_{0}}{r}\right)^{n}-n\left(\frac{r_{0}}{r}\right)^{m}\right]$ & $\mathrm{E}_{0}, \mathrm{n}, \mathrm{m}, \mathrm{r}_{0}$ & 0.2 & 12.0 & 6.0 & 3.37 \\
\hline
\end{tabular}

Table 2b. The Potential and Parameters for the Atomic Pair C-C

\begin{tabular}{|c|c|c|c|c|c|c|}
\hline Atomic pair & Potential & Functional Form & Parameters & $\mathrm{A}, \mathrm{kJ} / \mathrm{mol}$ & $\rho, \AA$ & $\mathrm{C}, \AA^{6}$ \\
\hline C-C & buck & $U(r)=A \exp \left(-\frac{r}{\rho}\right)-\frac{C}{r^{6}}$ & $\mathrm{~A}, \rho, \mathrm{C}$ & 369743.0 & 0.28 & 2576.2 \\
\hline
\end{tabular}

Table 2c. The Potential and Parameters for the Atomic Pair $\mathrm{C}-\mathrm{NO}_{3}$ and $\mathrm{NO}_{3}-\mathrm{NO}_{3}$

\begin{tabular}{|c|c|c|c|c|}
\hline Atomic pair & Potential & Functional Form & Parameters & $\boldsymbol{\varepsilon}, \mathbf{k J} / \mathbf{m o l}$ \\
\hline \hline $\mathrm{C}-\mathrm{NO}_{3}$ & $\mathrm{lj}$ & $U(r)=4 \varepsilon\left[\left(\frac{\sigma}{r}\right)^{12}-\left(\frac{\sigma}{r}\right)^{6}\right]$ & $\varepsilon, \sigma$ & 0.41838 \\
& & $\ldots$ & & \\
\hline $\mathrm{NO}_{3}-\mathrm{NO}_{3}$ & $\mathrm{lj}$ & $\varepsilon, \sigma$ & 0.14 \\
\hline
\end{tabular}

(a)

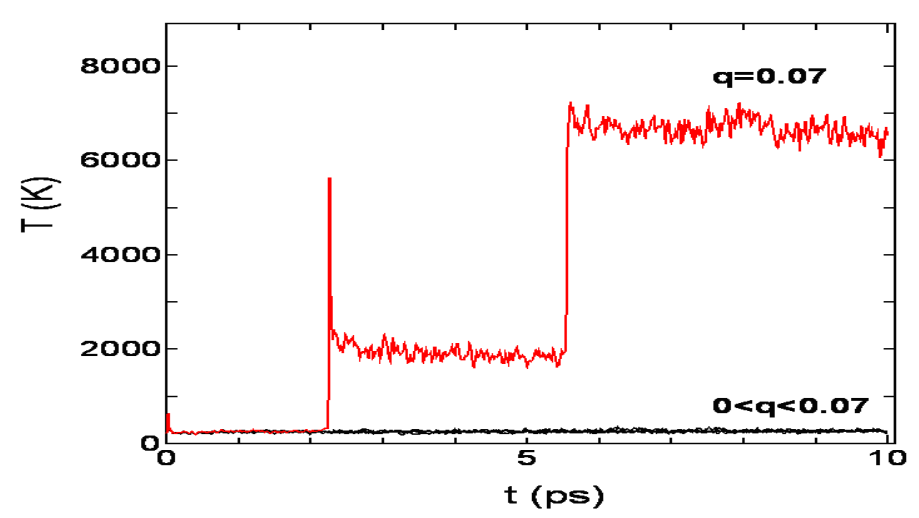

(b)

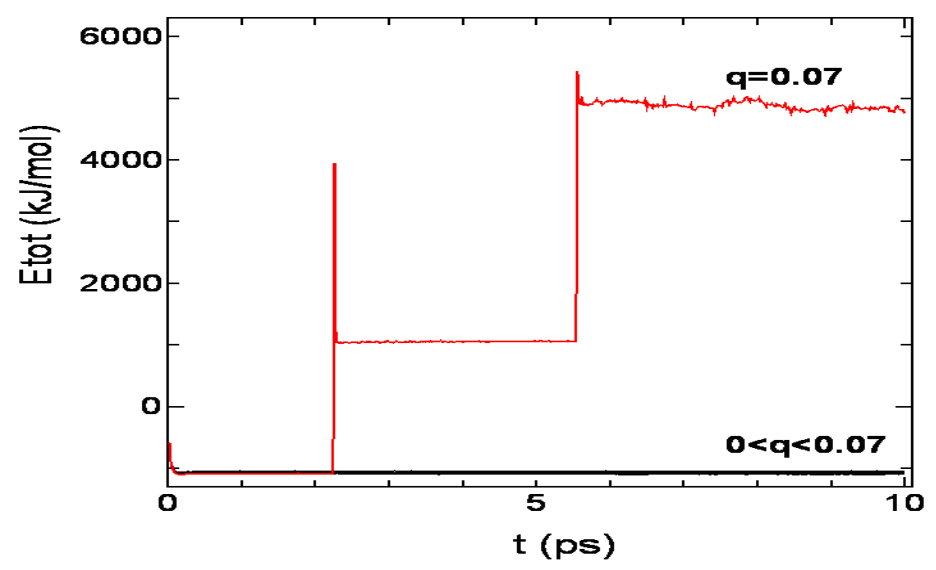

Fig. (3). The temperature (a) and total energy (b) of the system at different charge numbers of the solvent particles. 
microcanonical (nve) ensemble was used for the system simulated. The intermolecular chemical bonds were estimated on the basis of the Shake algorithm with an accuracy of $10^{-8}$. The Ewald summation with a convergence parameter of $10^{-6}$ was used for the calculation of electrostatics forces in the periodic system.

\section{RESULTS AND DISCUSSIONS}

Threshold Behavior of Temperature and Total Energy

For relaxed configurations of biphenyl molecules with the $\mathrm{HNO}_{3}$ solvent, we performed a series of the MD calculations under a constant temperature and environmental setup, but varying the solvent charge number:

Table 3. The Values of the System's Temperature and Total Energy for $q>0.07$

\begin{tabular}{|c|c|c|c|}
\hline & Region I $(\mathbf{0}<\mathbf{t}<\mathbf{2 . 2} \mathbf{p s})$ & Region II $(2.2$ ps $<\mathbf{t}<\mathbf{5 . 6}$ ps $)$ & Region III $(\mathbf{5 . 6}$ ps $<\mathbf{t}<\mathbf{1 0}$ ps $)$ \\
\hline \hline $\mathrm{T}(\mathrm{K})$ & 250 & 2000 & 6700 \\
\hline $\mathrm{E}(\mathrm{kJ} / \mathrm{mol})$ & -1090 & 1050 & 4900 \\
\hline
\end{tabular}

(a) $\mathrm{q}<\mathrm{q}_{\mathrm{cr}}$
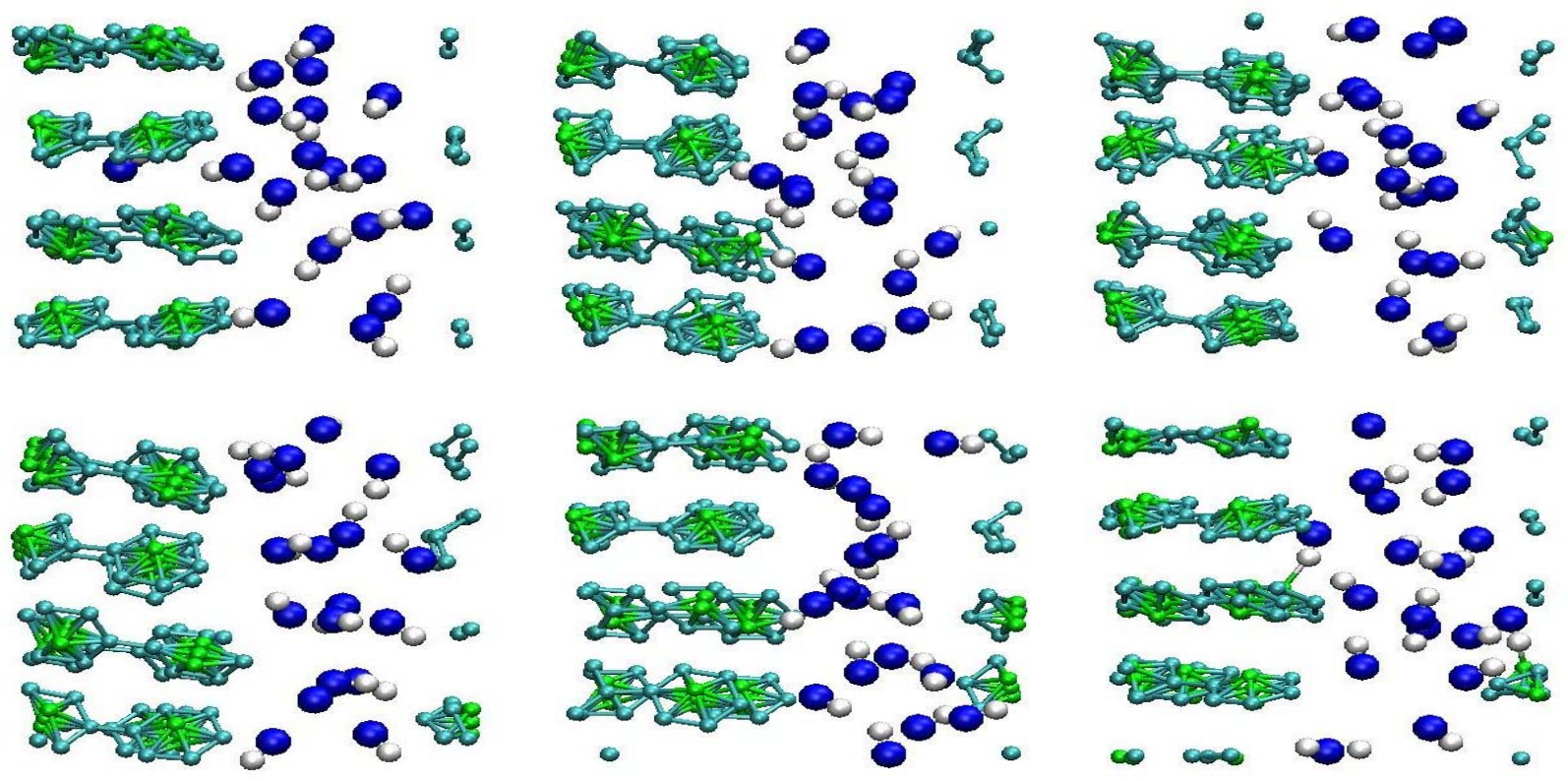

(b) $\mathrm{q}>\mathrm{q}_{\mathrm{cr}}$
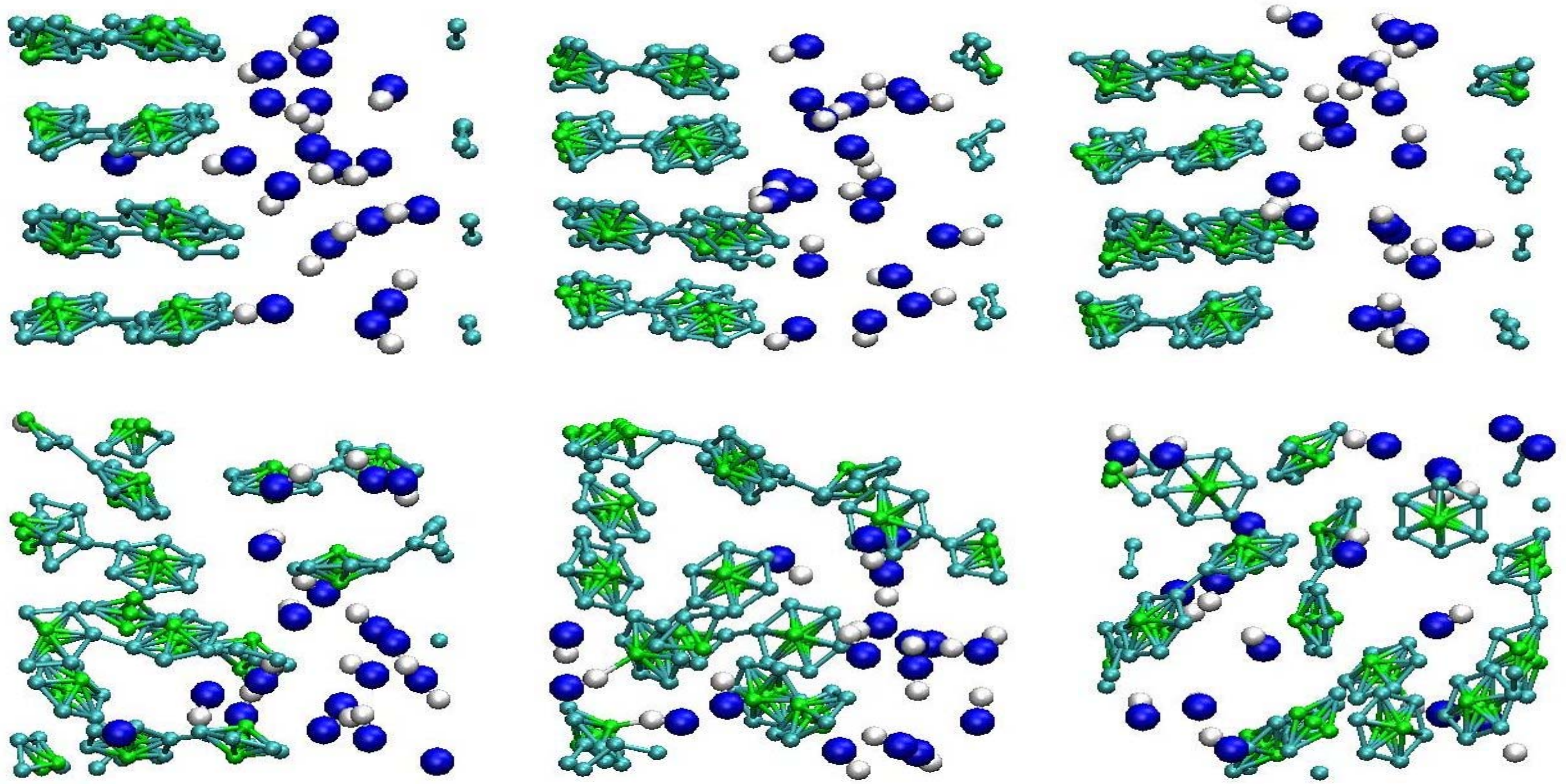

Fig. (4). Six consequent MD configurations of biphenyl in acid solvent are presented: $q=0.05\left(\mathrm{q}<\mathrm{q}_{\mathrm{cr}}\right)(\mathbf{a})$; $\mathrm{q}=0.07$ ( $\left.\mathrm{q}>\mathrm{q}_{\mathrm{cr}}\right)$ (b). The snapshots correspond to the time moments $\mathrm{t}=0,1,2,3,5$ and $10 \mathrm{ps}$ (left to right and top to bottom). 
$\mathrm{q}=-\mathrm{q}\left(\mathrm{NO}_{3}\right)=+\mathrm{q}\left(\mathrm{H}^{+}\right)=0,0.01,0.02, \ldots, 1$.

It should be stressed that the charge number q was varied for the solvent atoms only; the biphenyl's atomic charges were fixed as shown in Table $\mathbf{1}$. Thus, for the solvent atoms, we simulated several charge numbers $\mathrm{q}$ so that it took all possible values in the interval $[0,1]$. Of course, quantum chemistry or $a b$ initio calculations can provide a unique and exact value of q corresponding to its equilibrium state or phase diagram. However, the advantage of the present MD simulation approach is that one can take into account the whole range of the possible values of the charge number, thereby enabling a data analysis of a broad spectrum of possible experimental realizations.

The simulation results on the dynamics of the system's temperature and total energy are presented in Fig. $(\mathbf{3 a}, \mathbf{b})$. It is seen that for all charge numbers in the interval $0<\mathrm{q}<0.07$, the temperature and total energy vary near their relaxed values $\mathrm{T}=250 \mathrm{~K}$ and $\mathrm{E}=-1090 \mathrm{~kJ} / \mathrm{mol}$, respectively. However, at the values $\mathrm{q}=-\mathrm{q}\left(\mathrm{NO}_{3}{ }^{-}\right)=+\mathrm{q}\left(\mathrm{H}^{+}\right)>0.07$, the temperature and total energy change sharply, indicating that an essential phase reconstruction occurs in the molecular system. Namely, for the values $q>0.07$, we observe three distinct regions of the temperature - energy values as follows (see Table 3):

(1) in the interval of $0<\mathrm{t}<2.2 \mathrm{ps}$, the temperature and total energy oscillate around the relaxed values $\mathrm{T}=250 \mathrm{~K}$ and $\mathrm{E}=-1090 \mathrm{~kJ} / \mathrm{mol}$, respectively; in the interval of $2.2 \mathrm{ps}<\mathrm{t}<5.6 \mathrm{ps}$, the first jump of the temperature up to $\mathrm{T}=2000 \mathrm{~K}$ and total energy up to $\mathrm{E}=1050 \mathrm{~kJ} / \mathrm{mol}$ occurs;

(3) in the interval of $5.6 \mathrm{ps}<\mathrm{t}<10 \mathrm{ps}$, the second jump of the temperature up to $\mathrm{T}=6700 \mathrm{~K}$ and total energy up to $E=4900 \mathrm{~kJ} / \mathrm{mol}$ occurs.

\section{The Structural Configurations of the Biphenyl Molecule in the $\mathrm{HNO}_{3}$ Solvent}

From biphenyl's temperature - energy behavior, as presented in the previous section, we found the critical value of the charge number, namely, $\mathrm{q}_{\mathrm{cr}}=0.06$, which defines the temperature - energy modes in the system. To illustrate the biphenyl structural changes at different temperature - energy modes that are shown in Fig. (3), we compared the system configuration snapshots for the charge numbers $q=0.05$ $\left(\mathrm{q}<\mathrm{q}_{\mathrm{cr},}\right.$, Fig. 4a) and $\mathrm{q}=0.07\left(\mathrm{q}>\mathrm{q}_{\mathrm{cr}}\right.$, Fig. $\left.4 \mathrm{~b}\right)$. It is seen that at $\mathrm{q}=0.05$ (Fig. (4a)), the structure of the biphenyl complex kept its regular shape, both as a whole molecular complex and as a separate molecule. In contrast, at $\mathrm{q}=0.07$ (Fig. (4b)), biphenyl's regular structure broken sharply, and we observed the destruction of the biphenyl complex. The destruction first occurs for the whole complex; then, for each molecule separately. Such behavior, as shown in Figs. (4a, b), fully correlates with the results shown in Fig. (3). Thus, it is seen that a threshold for the temperature - energy dynamics has to exist (regions II and III, Fig. 3), where the system's temperature - energy jumps occur twice. At the first jump,

(a)

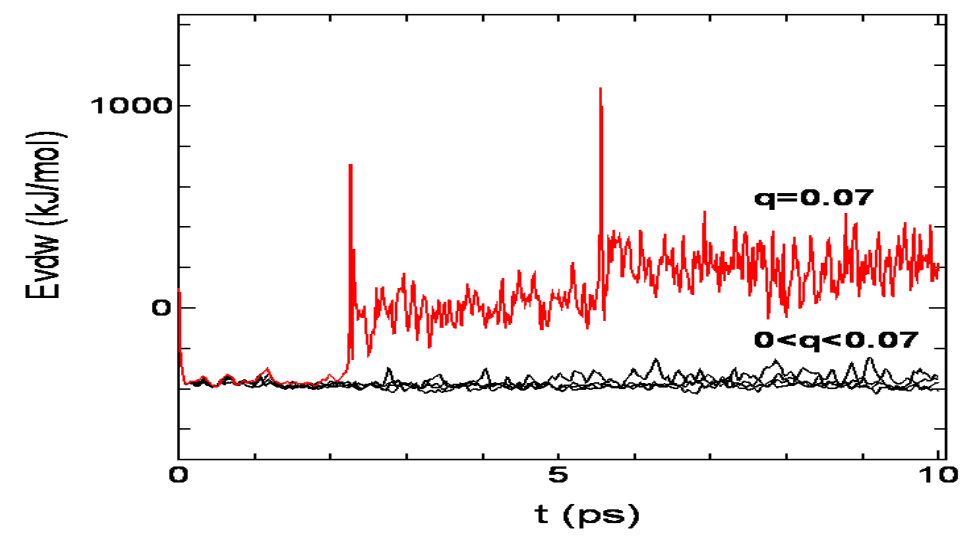

(b)

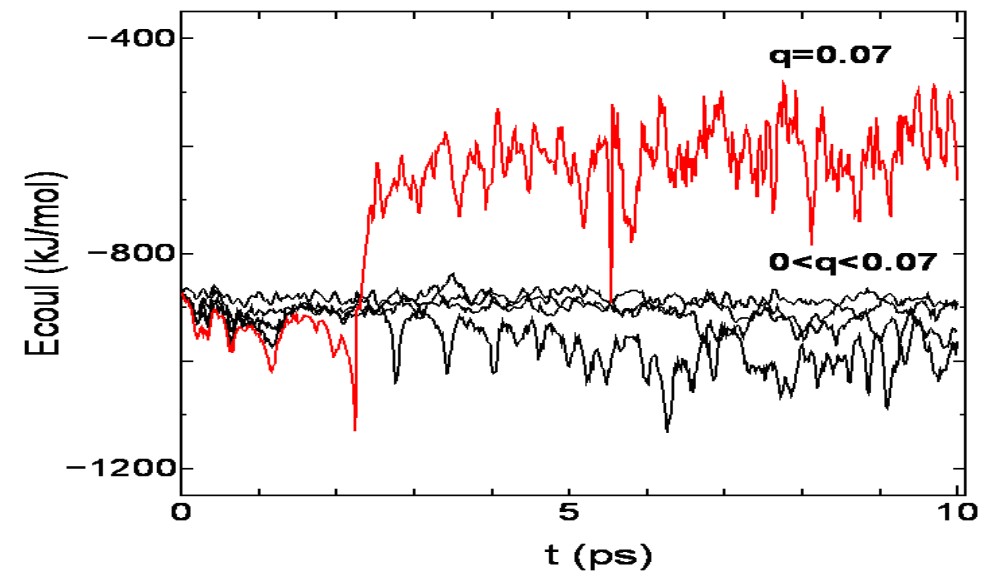

Fig. (5). The van der Waals (a) and electrostatic (b) energies at different values of the charge numbers of solvent particles. 
the temperature sharply changes from a relaxed value of 250 $\mathrm{K}$ up to $2000 \mathrm{~K}$; in this region, we observe the disruption of the regular structure of biphenyl as a whole complex. At the second jump, the system temperature sharply changes from $2000 \mathrm{~K}$ up to $6700 \mathrm{~K}$; in this region, we observe the destruction of each biphenyl as a separate fragment.

Next, the energy diagrams of the non-bonded (van der Waals, $\mathrm{E}_{\mathrm{vdW}}$, and electrostatic, $\mathrm{E}_{\mathrm{coul}}$ ) along with the bonded (angular, $\mathrm{E}_{\mathrm{ang}}$, and dihedral, $\mathrm{E}_{\mathrm{dih}}$ ) interactions, which are presented in Figs. $(\mathbf{5 a}, \mathbf{b})$ and $(\mathbf{6 a}, \mathbf{b})$, correlate well with the threshold behavior as discussed above.

In summary, with regard to the chemical or biochemical phenomena involving biphenyl, the important issues are bond formation and destruction of various specific forms. It is well known that the biphenyl molecule is able to take part in a huge number of intermolecular interactions, and, hence, is able to form all types of the chemical bonds - covalent, ionic, hydrogen, or van-der-Waals ones [1-9,12-14]. Our MD simulation results illustrate a nontrivial picture of the interaction between biphenyl and the $\mathrm{HNO}_{3}$ solvent namely, the threshold behavior of biphenyl's structural changes in an acidic environment. Such a threshold nature is determined by the flexibility of the solvent atomic charges.

\section{ACKNOWLEDGEMENTS}

This work has been performed under joint collaboration agreements with Daresbury Laboratory, UK, and Keio University, Japan. We would like to thank Prof. William Smith for the software support.

\section{REFERENCES}

[1] Gener, I.; Ginestet, G.; Buntinx, G.; Bremard C. Sorption of biphenyl in NaX zeolites. Phys. Chem. Chem. Phys., 2000, 2(8), 1855-1864.

[2] Salsbury, F.R. Jr.; Crowder, M.W.; Kingsmore, S.F.; Huntley, J.J. Molecular dynamics simulations of the metallo-beta-lactamase from Bacteroids fragilis in the presence and absence of a tightbinding inhibitor. J. Mol. Model., 2009, 15(2), 133-145.
[3] Tigini, V.; Prigione, V.; Toro, S.D.; Fava, F.; Varese, G.C. Isolation and characterization of polychlorinated biphenyl (PCB) degrading fungi from a historically contaminated soil. Microb. Cell Fact., 2009, 8(5), 1-14.

[4] Vasilyeva, G.K.; Strijakova, E.R. Bioremediation of soil and sediment contaminated by polychlorobiphenils. Microbiology, 2007, 76(6), 639-653.

[5] Szejtli, J. Utilization of cyclodextrins in industrial products and processes. J. Mater. Chem., 1997, 7, 575-587.

[6] Manunza, B.; Deianna, S.; Pintore, M.; Gessa C. Structure and internal motion of solvated beta-cyclodextrine: a molecular dynamics study. J. Mol. Struct.: THEOCHEM, 1997, 419(1), 133137(5).

[7] Saegner, W.; Jacob, J.; Gessler, K.; Steiner, T.; Hoffman, D.; Sanbe, H.; Koizumi, K.; Smith, S.M.; Takaha, T. Structures of the common cyclodextrins and their larger analogues-beyond the doughnut. Chem. Rev., 1998, 98(5), 1787-1802.

[8] Caudle, W.M.; Richardson, J.R.; Delea, K.C.; Guillot, T.S.; Wang, M.; Pennell, K.D.; Miller, G.W. Polychlorinated biphenyl-induced reduction of dopamine transporter expression as a precursor to Parkinson's disease-associated dopamine toxicity. Toxicol. Sci., 2006, 92(2), 490-499.

[9] Strazzolini, P.; Verado, G.; Gorassini, F.; Giumanini G.A. Orientation effect of side chain substituents in aromatic substitution. Induced ortho nitration. Bull. Chem. Soc. Jpn., 1995, 68(04), 1155-1161.

[10] Smith, W.; Forester, T. DL_POLY_2.0: A general-purpose parallel molecular dynamics simulation package, J. Mol. Graph., 1996, 14(3), 136-141.

[11] Smith, W.; Forester, T.R.; Todorov, I.T. The DL poly 2 user manual, stfc Daresbury Laboratory Daresbury, Warrington WA4 4AD Cheshire, UK, Version 2.19, April 2008

[12] Suzuki, H.; Takeuchi, T.; Mori, T. Ozone-mediated nitration of phenylalkyl ethers, phenylacetic esters, and related compounds with nitrogen dioxide. The highest ortho substitution observed in the electrophilic nitration of arenes. J. Org Chem., 1996, 61(17), 5944-5947.

[13] Misra, A.K.; Agnihotri, G. Nitric acid mediated oxidative transformation of thiols to disulfides. Synth. Commun., 2004, 34(6), 1079-1085.

[14] Giumanini, A.G.; Geatti, P.; Verardo, G. A convenient ar-S laboratory experiment avoiding the use of sulfuric acid: the nitration of diphenylmethane in $\mathrm{CH}_{2} \mathrm{Cl}_{2}$. Chem. Educ., 2002, 7(1), 27-32.

(C) Kholmurodov et al.; Licensee Bentham Open.

This is an open access article licensed under the terms of the Creative Commons Attribution Non-Commercial License (http://creativecommons.org/licenses/by-nc/3.0/) which permits unrestricted, non-commercial use, distribution and reproduction in any medium, provided the work is properly cited. 